\title{
Function of mitochondrial pyruvate carriers in hepatocellular carcinoma patients
}

\author{
XIANGMING MA, YUNLONG CUI, HONGYUAN ZHOU and QIANG LI
}

Department of Hepatobiliary Surgery, Tianjin Medical University Cancer Institute and Hospital, Key Laboratory of Cancer Prevention and Therapy, National Clinical Research Center for Cancer, Tianjin 300060, P.R. China

Received October 9, 2016; Accepted July 9, 2017

DOI: $10.3892 / \mathrm{ol} .2018 .8466$

\begin{abstract}
Mitochondrial pyruvate carriers (MPC) have been identified as a critical component of energy metabolism in the cancer cells of multiple malignant tumor types. The aim of the present study was to investigate the association between the expression of MPC1 and MPC2 and the prognosis of patients with hepatocellular carcinoma (HCC). A total of 85 formalin-fixed paraffin-embedded HCC tissues were assessed using immunohistochemistry. A further 20 fresh pathological specimens, including cancer and adjacent normal liver tissues from patients who had undergone a hepatectomy, were analyzed using western blotting and reverse transcription-quantitative polymerase chain reaction. The relative expression of MPC1 and MPC2 was quantified using Image-Pro Plus software, and the association between MPC expression and clinical outcomes was analyzed by Student's t-test. MPC1 and MPC2 protein expression was significantly downregulated in $\mathrm{HCC}$, but no association was identified between the expression of MPC1 or MPC2 and the clinicopathological characteristics of the patients. MPC1 mRNA levels were decreased in each cancer sample, while a mixture of increased and decreased MPC2 mRNA levels observed in the HCC samples. Multivariate regression analysis indicated that the protein level and the microvascular invasion of MPC1 were positively associated with the recurrence of $\mathrm{HCC}(\mathrm{P}=0.000$ and $\mathrm{P}=0.017$, respectively). MPC1 may therefore serve as an attractive biomarker for the identification of patients with HCC at a high risk of recurrence following curative resection.
\end{abstract}

Correspondence to: Professor Qiang Li, Department of Hepatobiliary Surgery, Tianjin Medical University Cancer Institute and Hospital, Key Laboratory of Cancer Prevention and Therapy, National Clinical Research Center for Cancer, Huan-Hu-Xi Road, He Xi, Tianjin 300060, P.R. China

E-mail: liqiang4016@yahoo.com

Key words: hepatocellular carcinoma, relapse, prognostic value, mitochondrial pyruvate carriers

\section{Introduction}

Hepatocellular carcinoma (HCC) is the most common histological type in the majority of patients with liver cancer, with increasing morbidity and mortality rates. Over the last few decades, management of HCC, including surveillance programs, diagnostic capacity and effective curative treatment methods, have substantially improved, thereby improving patient survival times and quality of life (1). However, the high rate of 5-year relapse remains an important problem in postoperative patients with HCC. In certain Asian countries, the 5-year cumulative rate of recurrence following primary hepatic resection may be as high as $70-100 \%(2,3)$. Although previous studies have provided evidence that certain biomarkers may be used to predict the recurrence of HCC (4-6), little has been identified regarding the crucial biomarkers required to guide the target of potential treatments and to prevent HCC recurrence.

It is now known that the majority of cancerous cells undergo bioenergetic reprogramming, switching the maximal pyruvate metabolism from mitochondrial oxidative phosphorylation to cytoplasm glycolysis, in order to support neoplastic proliferation, a process known as the 'Warburg effect' (7). During the conversion of tumor energy metabolism, functional mitochondria are essential for the viability of cancer cells (8). A few decades ago, studies reported that the amount of pyruvate transportation into the mitochondria and its utilization were significantly decreased, and that the malfunction of mitochondrial pyruvate carrier (MPC) activity was associated with the proliferation of tumor cells $(9,10)$. In line with this, using a specific inhibitor of MPC slightly enhanced tumor growth (11).

MPC, the molecular identification and purification of which were achieved in $2012(12,13)$, is a protein complex comprised of MPC1 (also known as BRP44L) and MPC2 (also known as BRP44) in humans. More recently, depletion or extremely low levels of MPC1 protein were revealed to be common features of multiple malignant cancer types and indicators of a poorer prognosis (14). Studies published thus far demonstrate that as a linker of glycolysis and intra-mitochondrial pyruvate metabolism, MPC is likely to have marked effects on the phenotypes of tumor metabolism and proliferation. As mitochondria are highly abundant within liver cells, the present study proposes that MPC may be associated with HCC and thus may serve a vital role in its initiation and progression. 
The present study aimed to evaluate the association between MPC1 and MPC2 and the clinicopathological parameters and prognosis of $\mathrm{HCC}$, and therefore to provide a potential biomarker for the recurrence and prognosis of HCC.

\section{Patients and methods}

Sample collection. The present study was approved by the Ethics Committee of the Cancer Institute of Tianjin Medical University Cancer Institute and Hospital (Tianjin, China). Written informed consent was obtained prior to participation in the study. A total of 85 patient samples were used for immunohistochemistry (primary HCC tissues and their adjacent non-cancerous tissues). The samples were obtained from patients who had undergone a curative liver resection between January 2011 and December 2012, following a primary histopathologically confirmed HCC diagnosis. The relevant clinicopathological characteristics of the patients with HCC are presented in Table I. Follow-up occurred between the date of the hepatectomy and November 2015. Fresh hepatocarcinoma samples and para-carcinoma tissue were placed in liquid nitrogen immediately when the specimens were isolated between May 2015 and August 2015, to be used for mRNA and western blot analysis. Recurrence-free survival (RFS) was determined following a radiologically evident diagnosis of recurrence (using computed tomography and/or magnetic resonance imaging). Overall survival (OS) was the percentage of patients who survived since curative liver resection.

Immunohistochemistry. All tissue samples were fixed with $10 \%$ neutral formalin, embedding into paraffin sections $(4 \mu \mathrm{m})$ after $24 \mathrm{~h}$ and were subsequently deparaffinized with dimethylbenzene and rehydrated through a concentration gradient of ethanol, prior to antigen retrieval in a pressure cooker $(\mathrm{pH}=6.0$ sodium citrate acid repair solution, $121^{\circ} \mathrm{C}$ for $5 \mathrm{~min}$. Samples were then washed in PBS 3 times, and endogenous peroxidase activity was inactivated in $3 \%$ hydrogen peroxide solution for $10 \mathrm{~min}$ at room temperature. Sections were incubated with an anti-BRP44L antibody (cat no., ab74871; dilution, 1:50; Abcam, Cambridge, UK) and an anti-BRP44 antibody (cat no., ab111380; dilution, 1:50; Abcam) overnight at $4^{\circ} \mathrm{C}$. Horseradish peroxidase-tagged antibody (cat no., PV-9000; ready-to-use; Beijing Zhongshan Jinqiao Biotechnology Co., Ltd., Beijing, China) was added and incubated for $30 \mathrm{~min}$ at room temperature, followed by positive staining with diaminobenzidine $(0.05 \%)$ for $1 \mathrm{~min}$ at room temperature, and counterstaining with hematoxylin $(0.1 \%)$ for $1 \mathrm{~min}$ at room temperature. The slides were observed and imaged under a positive optical microscope. The relative protein expression was quantified by Image-Pro Plus version 5.0 software (Media Cybernetics Inc., Rockville, MD, USA) and defined as follows: Density mean=density sum/area sum (15).

Western blot analysis. The proteins in the cells and tissues were lysed on ice in a radioimmunoprecipitation assay lysis buffer (cat no., P0013-B; Beyotime Institute of Biotechnology, Haimen, China), protein concentration was determined using a BCA Protein Assay kit (Pierce; Thermo Fisher Scientific, Inc., Waltham, MA, USA) and equal amounts of total protein $(30 \mu \mathrm{g})$ were separated by SDS-PAGE (12\% gels) and transferred onto polyvinylidene difluoride membrane. The membranes were blocked with 5\% skimmed milk followed by incubation with the specific primary antibodies and $\beta$-actin control antibody (cat no., sc-47778; dilution, 1:1,000; Santa Cruz Biotechnology, Inc., USA), anti-BRP44 L antibody (cat no., ab74871; dilution, 1:500; Abcam) and anti-MPC2 polyclonal antibody (cat no., 20049-1-AP; dilution, 1:500; ProteinTech Group, Inc., Chicago, IL, USA), overnight at $4^{\circ} \mathrm{C}$. The immunoreactivity signals were visualized by enhanced chemiluminescence reagents according to the manufacturer's protocols (EMD Millipore, Billerica, MA, USA) following incubation with a horseradish peroxidase (HRP)-conjugated goat anti-mouse immunoglobulin G (cat no. 10004302-1) and HRP-conjugated goat anti-rabbit $\operatorname{IgG}$ (cat no A21020) secondary antibodies (both, 1:5,000; Beijing Zhongshan Jinqiao Biotechnology Co., Ltd., Beijing, China), for $60 \mathrm{~min}$ at room temperature.

$R N A$ isolation, reverse transcription-polymerase chain reaction ( $R T-P C R)$, and quantitative PCR ( $q P C R)$. Total RNA was extracted from tissues using TRIzol reagent (Ambion, Thermo Fisher Scientific, Inc.) prior to being reverse transcribed into cDNA using PrimeScript RT Master mix (Takara Biotechnology Co., Ltd., Dalian, China), according to the manufacturer's protocols. qPCR was performed using SYBR Premix Ex Taq ${ }^{\mathrm{TM}}$ II (Takara Biotechnology Co., Ltd.) on a CFX96 Real-Time PCR Detection system (Bio-Rad Laboratories, Inc., Hercules, CA, USA). Thermocycling conditions were as follows: $95^{\circ} \mathrm{C}$ for $30 \mathrm{sec}, 45$ cycles of melting at $95^{\circ} \mathrm{C}$ for $5 \mathrm{sec}$, followed by $60^{\circ} \mathrm{C}$ for $30 \mathrm{sec}$. The mRNA expression level was normalized to GAPDH. The forward and reward primer sequences were as follows: GAPDH forward, 5'-GAA GGTGAAGGTCGGAGTC-3' and reverse, 5'-GAAGATGGT GATGGGATTTC-3'; MPC1 forward, 5'-CGCGTTGGTGCG GAAAGCG-3' and reverse, 5'-GGCAAATGTCATCCGCCC ACTGA-3'; and MPC2 forward, 5'-TACCACCGGCTCCTC GATAAA-3' and reverse, 5'-TATCAGCCAATCCAGCAC ACA-3'. Quantification of the MPC1, MPC2 and GAPDH bands were calculated using the $2^{-\triangle \Delta C q}$ method (16). The MPC1 and MPC2 mRNA expression ratio of non-cancerous tissue to tumor tissues was analyzed using $\log _{2}$.

Statistical analysis. Statistical analyses were performed by IBM SPSS Statistics 22 (IBM Corp., Armonk, NY, USA). Statistical analyses of continuous variables are expressed as the mean \pm standard deviation. Pearson's correlation analysis was used to estimate the association between MPC1 and MPC2, at mRNA and protein level. Student's t-test was used to analyze the association between expression level and continuous variables, while the $\chi^{2}$ test was used for categorical variables of clinicopathological characteristics. The Kaplan-Meier method was used to estimate the RFS rates, and significant differences was assessed using the log-rank test. Univariate and multivariate survival analyses were performed using the Cox's proportional hazards model. $\mathrm{P}<0.05$ was considered to indicate a statistically significant difference.

\section{Results}

Expression of MPC1 and MPC2 in HCC and paired adjacent hepatic tissues. To examine whether MPC1 or MPC2 were 
Table I. Association between MPC protein expression in tumor tissue and clinicopathological parameters in hepatocellular carcinoma patients.

\begin{tabular}{|c|c|c|c|c|c|c|}
\hline \multirow[b]{2}{*}{ Clinical characteristics } & \multicolumn{3}{|c|}{ MPC1 } & \multicolumn{3}{|c|}{ MPC2 } \\
\hline & High $(n=43)$ & Low $(n=42)$ & P-value & High $(n=42)$ & Low $(n=43)$ & P-value \\
\hline Age, year & $54.8 \pm 9.5$ & $54.7 \pm 11.2$ & 0.426 & $55.7 \pm 10.9$ & $53.9 \pm 9.8$ & 0.386 \\
\hline Gender (male/female) & $33 / 10$ & $37 / 5$ & 0.255 & $33 / 9$ & $37 / 6$ & 0.407 \\
\hline $\operatorname{ALT}(\mathrm{U} / \mathrm{l})$ & $37.4 \pm 25.5$ & $37.0 \pm 19.0$ & 0.131 & $34.1 \pm 21.7$ & $37.2 \pm 23.1$ & 0.626 \\
\hline $\operatorname{AST}(\mathrm{U} / \mathrm{l})$ & $40.6 \pm 27.1$ & $37.6 \pm 27.0$ & 0.864 & $39.5 \pm 32.1$ & $37.8 \pm 21.4$ & 0.507 \\
\hline TBIL $(\mu \mathrm{mol} / \mathrm{l})$ & $17.0 \pm 10.0$ & $19.6 \pm 12.9$ & 0.999 & $19.6 \pm 14.2$ & $17.2 \pm 8.2$ & 0.120 \\
\hline $\operatorname{ALB}(g / l)$ & $46.3 \pm 4.1$ & $43.5 \pm 6.6$ & 0.187 & $44.0 \pm 6.6$ & $45.8 \pm 4.4$ & 0.431 \\
\hline PT, sec & $11.2 \pm 1.1$ & $11.8 \pm 3.1$ & 0.296 & $11.7 \pm 3.2$ & $11.4 \pm 1.2$ & 0.441 \\
\hline PLT, $x 10^{9} / 1$ & $158.0 \pm 69.8$ & $167.9 \pm 48.9$ & 0.473 & $162.6 \pm 80.4$ & $163.6 \pm 69.1$ & 0.411 \\
\hline $\operatorname{AFP}(>200 / \leq 200 \mathrm{ng} / \mathrm{ml})$ & $16 / 27$ & $15 / 27$ & 1.000 & $17 / 25$ & $14 / 29$ & 0.504 \\
\hline BCLC Stage (0-A/B-C) & $23 / 20$ & $15 / 27$ & 0.128 & $18 / 24$ & $20 / 23$ & 0.828 \\
\hline Tumor number $(1 />1)$ & $35 / 8$ & $39 / 3$ & 0.195 & $36 / 6$ & $38 / 5$ & 0.757 \\
\hline \multicolumn{7}{|l|}{ Tumor size } \\
\hline Maximum diameter $(>5 / \leq 5 \mathrm{~cm})$ & $16 / 27$ & $21 / 21$ & 0.278 & $20 / 22$ & $17 / 26$ & 0.515 \\
\hline Smallest diameter $(>3 / \leq 3 \mathrm{~cm})$ & $19 / 24$ & $24 / 18$ & 0.281 & $22 / 20$ & $21 / 22$ & 0.829 \\
\hline Differentiation (poor/moderate or well) & $16 / 27$ & $13 / 29$ & 0.649 & $14 / 28$ & $15 / 28$ & 0.880 \\
\hline Microvascular invasion (yes/no) & $23 / 20$ & $25 / 17$ & 0.663 & $23 / 19$ & $25 / 18$ & 0.828 \\
\hline Micrometastases (yes/no) & $14 / 29$ & $18 / 24$ & 0.375 & $17 / 26$ & $16 / 27$ & 0.824 \\
\hline
\end{tabular}

Data are presented as mean \pm standard deviation or $\mathrm{n}$. MPC, mitochondrial pyruvate carrier; ALT, alanine aminotransferase; AST, aspartate aminotransferase; TBIL, total bilirubin; ALB, albumin; PT, prothrombin time; PLT, platelet; AFP, $\alpha$-fetoprotein.

dysregulated in HCC, cancerous and adjacent non-cancerous tissues from patients with HCC were surgically obtained. MPC1 and MPC2 protein expression levels were examined in 85 tissues sections of patients with HCC using immunohistochemistry staining, and in fresh samples using western blotting. Immunohistochemistry staining indicated that MPC1 and MPC2 protein expression was markedly lower in the tumor tissues than that in the peritumoral liver tissues (Fig. 1A). The relative protein expression was quantified by Image-Pro Plus software, and the results demonstrated that MPC1 and MPC2 protein expression was markedly decreased in HCC tissues $(\mathrm{P}<0.001)$ compared with that in the adjacent non-cancerous counterparts. Furthermore, this difference was more evident in MPC1 protein expression than in MPC2 protein expression (Fig. 1B), and the same result was obtained using western blotting (Fig. 1C). Additionally, the MPC1 and MPC2 mRNA expression level in 20 fresh harvested tumor specimens and their peripheral normal liver tissues was analyzed using by reverse transcription PCR. In line with previous results (14), the present study observed that the mRNA level of MPC1 was decreased in all the tumor tissues (20/20), but that the mRNA level of MPC2 was inconsistently dysregulated; upregulated in $9 / 20$ and downregulated in 11/20 HCC tissues (Fig. 1D). Although the expression of MPC2 mRNA was not consistent with its protein expression, there was a positive statistical association between MPC1 and MPC2 mRNA expression and between MPC1 and MPC2 protein expression (Fig. 1E). These data suggest that there may be a difference in the regulation of MPC1 and MPC2 at the mRNA level, and the two may serve a different function in the occurrence and clinical significance of HCC.

MPC expression and association with clinicopathological parameters. Based upon the consistently reduced expression of MPC protein in HCC tissues, the clinical significance of MPC1 and MPC2 expression levels were assessed. The density means of MPC1 and MPC2 were calculated, and according to the median protein expression in the group of tumor tissues, patients were split into two groups (low expression and high expression). However, no significant association was identified between patients' clinicopathological characteristics and their MPC1 or MPC2 protein expression levels ( $\mathrm{P}>0.10$; Table I). Therefore, this led us to hypothesize that these clinical and pathological parameters may not be associated with cancer energy metabolism.

Prognostic potential of MPC. To further clarify the exact function of the MPC in HCC following hepatectomy, the associations between the protein level of MPC1 and MPC2 and their respective prognostic functions were analyzed. The low MPC1 expression group had a significantly lower 3-year RFS rate following surgery compared with the high expression group ( $\mathrm{P}<0.001 ; 21.4$ and $55.8 \%$, respectively; Fig. 2A). The low MPC1 expression group was also associated with a significantly $(\mathrm{P}=0.001)$ shorter 3 -year OS compared with the high expression group (41.9 and 78.6\%, respectively; Fig. 2A). However, no significant association was identified between either RFS or OS and MPC2 expression levels $(\mathrm{P}=0.254$ 

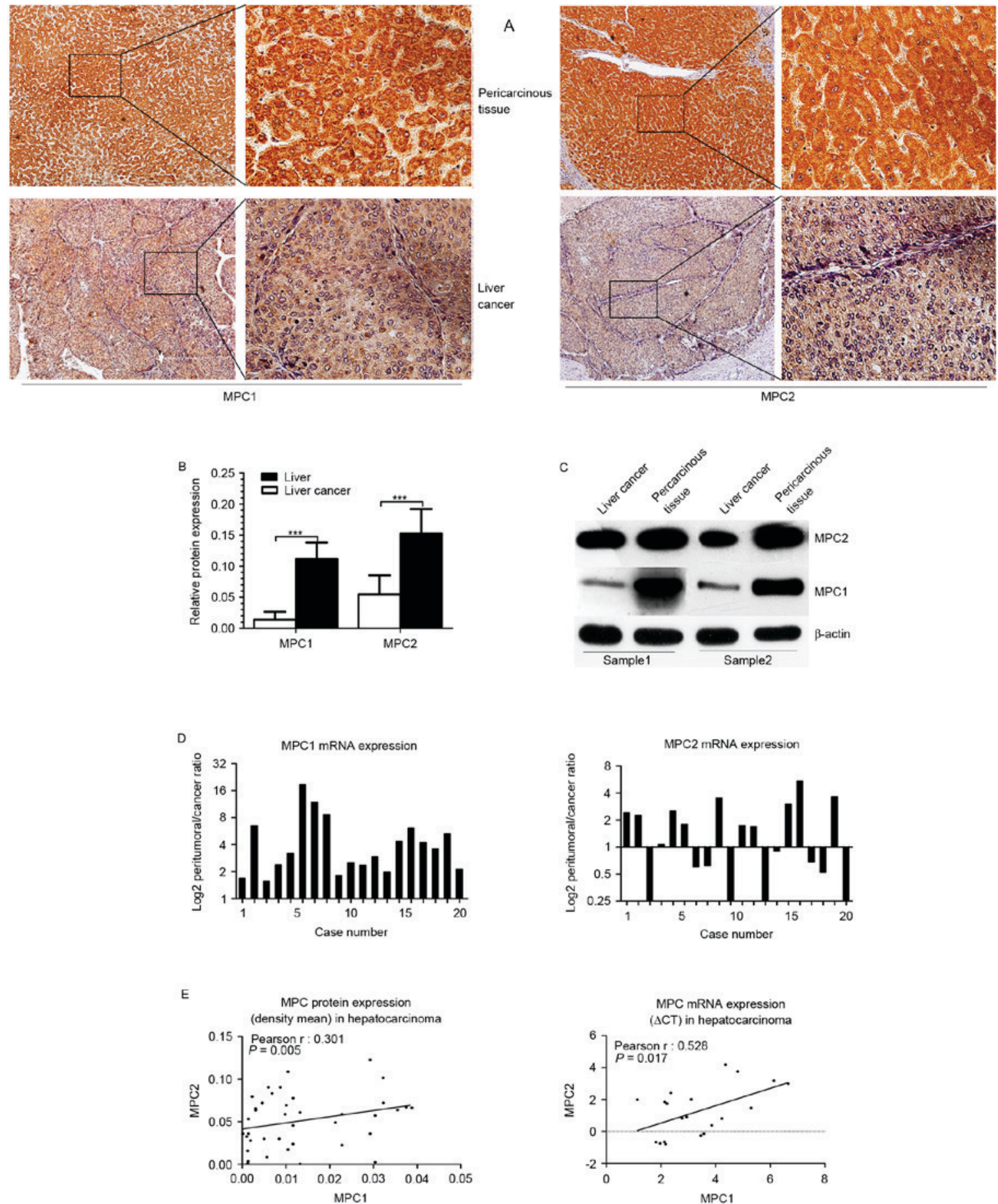

Figure 1. Expression of MPC in HCC tissues and adjacent liver tissues. (A) Expression of MPC1 (left) and MPC2 (right) protein in HCC tissues vs. adjacent uninvolved tissue was analyzed by immunohistochemical staining. (B) Relative expression of MPC1 and MPC2 protein was calculated by the density mean. Error bars represent means \pm standard deviation, ${ }^{* * * *} \mathrm{P}<0.001$. (C) Western blot analysis of MPC1 and MPC2 in HCC samples and paired normal tissues. (D) Expression of MPC1 (left) and MPC2 (right) mRNA. Plotted bar represents the log2 mRNA expression ratio of non-cancerous tissue to tumor tissues. (E) Co-expression analysis for MPC1 in HCC vs. MPC2. Protein expression is represented by the density mean value (left) and mRNA expression is represented by $\triangle \mathrm{Cq}$ (right). MPC, mitochondrial pyruvate carrier; HCC, hepatocellular carcinoma.

and $\mathrm{P}=0.452$, respectively; Fig. 2B). Univariate analysis revealed that Barcelona clinic liver cancer stage (17), and MPC1 expression were significant prognostic factors for RFS (Table II). Multivariate analysis using the Cox's proportional hazards model revealed that microvascular invasion [hazard ratio (HR), 2.115; 95\% confidence interval (CI), 1.143-3.913; $\mathrm{P}=0.017$; Table II] and MPC1 expression (HR, 3.773; 95\% CI, 2.113-6.737; $\mathrm{P}=0.000$; Table II) were independent recurrence risk factors in HCC patients who had undergone hepatectomy. These data demonstrate that a loss of MPC1 protein is strongly associated with cancer relapse and a poor prognosis.

\section{Discussion}

In humans, the two MPC complex proteins, MPC1 and MPC2, are required in order to facilitate pyruvate transport 
Table II. Univariate and multivariate analysis of the risk factors of hepatocellular carcinoma recurrence.

\begin{tabular}{|c|c|c|c|c|}
\hline \multirow[b]{2}{*}{ Variables } & \multicolumn{2}{|c|}{ Univariate analysis } & \multicolumn{2}{|c|}{ Multivariate analysis } \\
\hline & $\mathrm{HR}(95 \% \mathrm{CI})$ & P-value & $\mathrm{HR}(95 \% \mathrm{CI})$ & P-value \\
\hline Age $(>60 / \leq 60$ years $)$ & $1.096(0.594-2.023)$ & 0.769 & & \\
\hline Gender (male/female) & $0.677(0.318-1.439)$ & 0.311 & & \\
\hline $\operatorname{AFP}(>200 / \leq 200$ ng/ml $)$ & $0.690(0.396-1.201)$ & 0.190 & & \\
\hline BCLC stage $(0-\mathrm{A} / \mathrm{B}-\mathrm{C})$ & $0.497(0.282-0.878)$ & 0.016 & $1.288(0.657-3.622)$ & 0.461 \\
\hline Tumor number $(1 />1)$ & $1.080(0.487-2.397)$ & 0.850 & & \\
\hline \multicolumn{5}{|l|}{ Tumor size } \\
\hline Maximum diameter $(>5 / \leq 5 \mathrm{~cm})$ & $1.615(0.936-2.785)$ & 0.085 & $0.697(0.369-1.315)$ & 0.264 \\
\hline Smallest diameter $(>3 / \leq 3 \mathrm{~cm})$ & $1.228(0.713-2.117)$ & 0.459 & & \\
\hline Differentiation (poor/moderate or well) & $0.714(0.414-1.233)$ & 0.229 & & \\
\hline Microvascular invasion (presence/absence) & $1.732(0.983-3.053)$ & 0.057 & $2.115(1.143-3.913)$ & $0.017^{\mathrm{a}}$ \\
\hline Micrometastases (presence/absence) & $1.083(0.606-1.933)$ & 0.788 & & \\
\hline MPC1 expression (low/high) & $3.926(2.205-6.989)$ & 0.000 & $3.773(2.113-6.737)$ & $0.001^{\mathrm{a}}$ \\
\hline MPC2 expression (low/high) & $1.397(0.809-2.411)$ & 0.230 & & \\
\hline
\end{tabular}

${ }^{\mathrm{a}} \mathrm{P}<0.05$. HR, hazard ratio; 95\% CI, 95\% confidence interval; MPC, mitochondrial pyruvate carrier; AFP, $\alpha$-fetoprotein.
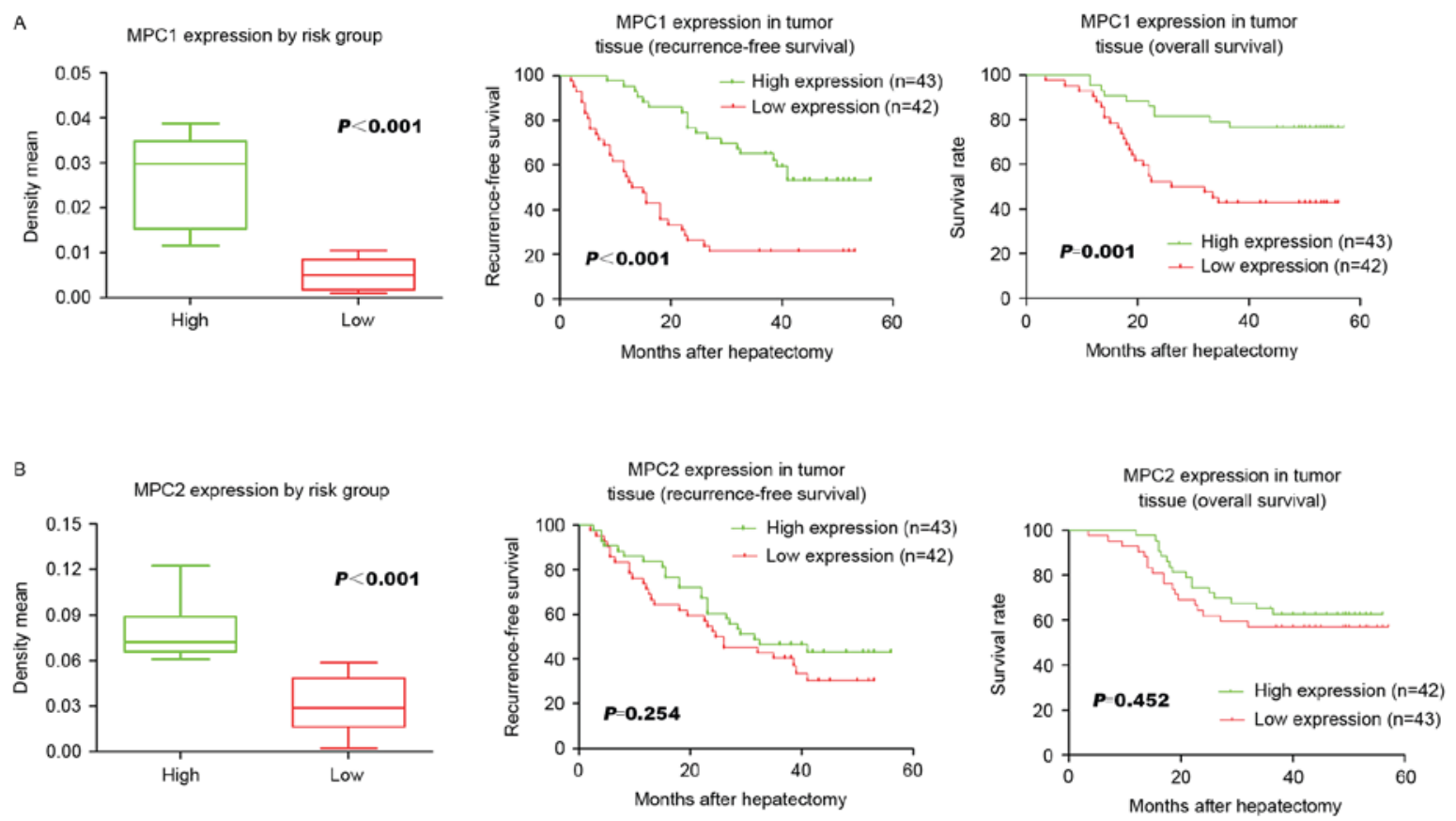

Figure 2. Association between MPC protein expression and the OS and RFS rates of patients with hepatocellular carcinoma. (A) A significant difference in MPC1 expression was observed with regard to RFS and OS. (B) MPC2 expression did not differ significantly with regard to RFS and OS. MPC, mitochondrial pyruvate carrier; OS, overall survival; RFS, recurrence-free survival.

across the mitochondrial membrane. Since its discovery, dysfunction of MPC has been observed in several cancer types, and in light of this, loss of the MPC has also been demonstrated to serve important functions in the development of tumors in liver cell lines, including normal rat liver, Ehrlich ascites tumor cells, Morris hepatoma 44 and Morris hepatoma 3924A cells $(10,14)$. However, the clinical significance of MPC expression in patients with HCC remains unknown. The present study identified that the protein expression of MPC1 and MPC2 was downregulated in HCC tumor tissues. Notably, the results indicated that low expression of these two proteins had no significant association with the clinical and pathological characteristics of the disease. Furthermore, the present study also demonstrated that low MPC1 may be an unfavorable prognostic biomarker, due to the fact that it is an independent risk factor for RFS and OS in patients with HCC who have undergone a hepatectomy. 
It is likely that mRNA expression is not a direct indication of protein expression, as the latter can be regulated not only at the transcription level, but also at the translational and turnover levels. The staining abundance of MPC1 and MPC2 protein expression in the present study differed significantly between tumor and non-cancerous tissues. The mRNA expression levels of MPC2 were associated with MPC1 in HCC tumor tissues, but unlike the consistent low expression of MPC1, the expression of MPC2 in hepatocellular carcinoma tissue was uneven. There are two potiential explanations for this discrepancy. One hypothesis is that certain MPC2 mRNAs are translated only once the blocking protein is removed by a certain signal. Another possibility is that MPC2 protein expression is regulated by certain modifications, including phosphorylation, acetylation, hydroxylation or changes in the space position (18). The mechanism(s) underlying the results of the present study remain unknown. Due to the fact that MPC is most abundant in the liver, it is indispensable for mitochondrial pyruvate import (19), and thus likely to constitute a critical feature of cancer cells. Further investigations are required to fill this gap, to survey and to evaluate if and how MPC modification affects its activity.

It should be noted that there are numerous factors, including tumor-, patient-, liver- and treatment-associated factors, in addition to the risk stratification schemes, which may be used to assess and improve the ability of clinicians to select patients and treatment methods, and a number of these have significant prognostic value and potential in improving patient outcomes $(20,21)$. The present study is the first to evaluate the association between MPC expression levels and clinical and tumor pathological characteristics, despite the fact that no association was observed. This lack of statistical significance may be due in part to the small sample size used $(n=85)$, the fact that other potentially associated factors may not have been included in the study or the fact that the tumor characteristics and clinical traits evaluated here have no association with the energy metabolism of tumor cells. Further investigation on the function of MPC in HCC is required in order for these mechanisms to be fully elucidated.

The high recurrence rate following $\mathrm{HCC}$ hepatectomy, which is a common occurrence posing a threat to patient outcomes, remains an unsolved problem (22). Regardless of any association between MPC and clinicopathological characteristics, however, it was observed that low MPC protein expression may serve as a definitive prognostic biomarker to monitor tumor relapse (RFS) over the time period following resection. In the present study, low MPC1 expression had a marked association with the risk of future recurrence and a shorter OS time. However, there was no statistically significant difference in either the RFS or OS rates observed between the HCC samples and their non-cancerous counterparts in the low MPC2 expression group. The present study indicated the high prognostic value of diminished MPC expression for determining the outcome of patients with HCC following resection.

Previous studies have demonstrated that inhibition of MPC activity has a profound impact on cell glucose and pyruvate metabolism, and that it induces glutaminolysis in the Krebs cycle $(11,23)$. Furthermore, various cancer cells appear to delete or suppress MPC expression and the study discussed herein (14) provide information regarding the function served by aberrant MPC activity in cancer metabolism. Additionally, gluconeogenesis is considered to be the reverse of glycolysis and is now recognized as a common hallmark of cancer. Gluconeogenesis is an important feature of hepatocytes, and pyruvate is the major substrate of gluconeogenesis. Silencing of liver MPC1 resulted in abolished hepatic MPC activity and a subsequent marked decrease in gluconeogenesis (24). Notably, in HCC tissues that have lost the ability to perform gluconeogenesis, focusing on the switch from glycolysis to gluconeogenesis may be an efficacious method for HCC treatment (25). Taken together, the results indicate that restoring the MPC function in patients with HCC may be a vital effective treatment. MPC activity has emerged to be inhibited by the insulin sensitizers, thiazolidinediones (26), and phosphodiesterase inhibitor Zaprinast (27). Drug targets to increase the efficacy of MPC remain elusive, although a previous study demonstrated that indirectly increasing the concentration of intracellular pyruvate can reverse the low MPC activity and cell respiration in various cell types (28).

In conclusion, MPC may serve a crucial role in repressing $\mathrm{HCC}$ recurrence by inhibiting pyruvate oxidative metabolism, and may be a promising attractive biomarker for patients with HCC following hepatectomy. Furthermore, the functions of MPC in liver glucose metabolism and in HCC onset or development require further investigation.

\section{Acknowledgements}

The authors would like to thank Dr Guo Hua, Dr Luo Yi, Dr Sun Bo, Dr Fu Hui, Dr Chen Lu, Miss Guo Piao and Miss Xi Qing from the Laboratory of Cancer Cell Biology of Tianjin Medical University Cancer Institute and Hospital for providing assistance with the experimental technique and for information support.

\section{References}

1. Fitzmorris P, Shoreibah M, Anand BS and Singal AK: Management of hepatocellular carcinoma. J Cancer Res Clin Oncol 141: 861-876, 2015.

2. El-Serag HB: Hepatocellular carcinoma. N Engl J Med 365: 1118-1127, 2011.

3. Takayama T: Surgical treatment for hepatocellular carcinoma. Jpn J Clin Oncol 41: 447-454, 2011.

4. Sengupta B and Siddiqi SA: Hepatocellular carcinoma: Important biomarkers and their significance in molecular diagnostics and therapy. Curr Med Chem 19: 3722-3729, 2012.

5. Schütte K, Schulz C, Link A and Malfertheiner P: Current biomarkers for hepatocellular carcinoma: Surveillance, diagnosis and prediction of prognosis. World J Hepatol 7: 139-149, 2015.

6. Saito Y, Shimada M, Utsunomiya T, Morine Y, Imura S, Ikemoto T, Mori H, Hanaoka J, Yamada S and Asanoma M: Prediction of recurrence of hepatocellular carcinoma after curative hepatectomy using preoperative Lens culinaris agglutinin-reactive fraction of alpha-fetoprotein. Hepatol Res 42: 887-894, 2012.

7. Hanahan D and Weinberg RA: Hallmarks of cancer: The next generation. Cell 144: 646-674, 2011.

8. Wallace DC: Mitochondria and cancer. Nat Rev Cancer 12: 685-698, 2012.

9. Eboli ML, Paradies G, Galeotti T and Papa S: Pyruvate transport in tumour-cell mitochondria. Biochim Biophys Acta 460: 183-187, 1977.

10. Paradies G, Capuano F, Palombini G, Galeotti T and Papa S: Transport of pyruvate in mitochondria from different tumor cells. Cancer Res 43: 5068-5071, 1983. 
11. Yang C, Ko B, Hensley CT, Jiang L, Wasti AT, Kim J, Sudderth J, Calvaruso MA, Lumata L, Mitsche M, et al: Glutamine oxidation maintains the TCA cycle and cell survival during impaired mitochondrial pyruvate transport. Mol Cell 56: 414-424, 2014

12. Herzig S, Raemy E, Montessuit S, Veuthey JL, Zamboni N Westermann B, Kunji ER and Martinou JC: Identification and functional expression of the mitochondrial pyruvate carrier. Science 337: 93-96, 2012.

13. Bricker DK, Taylor EB, Schell JC, Orsak T, Boutron A, Chen YC, Cox JE, Cardon CM, Van Vranken JG, Dephoure N, et al: A mitochondrial pyruvate carrier required for pyruvate uptake in yeast, Drosophila, and humans. Science 337: 96-100, 2012.

14. Schell JC, Olson KA, Jiang L, Hawkins AJ, Van Vranken JG, Xie J, Egnatchik RA, Earl EG, DeBerardinis RJ and Rutter J: A role for the mitochondrial pyruvate carrier as a repressor of the Warburg effect and colon cancer cell growth. Mol Cell 56: 400-413, 2014

15. Wang CJ, Zhou ZG, Holmqvist A, Zhang H, Li Y, Adell G and Sun XF: Survivin expression quantified by image pro-plus compared with visual assessment. Appl Immunohistochem Mol Morphol 17: 530-535, 2009.

16. Livak KJ and Schmittgen TD: Analysis of relative gene expression data using real-time quantitative PCR and the 2(-Delta Delta C(T)) method. Methods 25: 402-408, 2001.

17. Llovet JM, Brú C and Bruix J: Prognosis of hepatocellular carcinoma: The BCLC staging classification. Semin Liver Dis 19: 329-338, 1999

18. de Sousa Abreu R, Penalva LO, Marcotte EM and Vogel C: Global signatures of protein and mRNA expression levels. Mol Biosyst 5: 1512-1526, 2009.

19. Vigueira PA, McCommis KS, Schweitzer GG, Remedi MS, Chambers KT, Fu X, McDonald WG, Cole SL, Colca JR, Kletzien RF, et al: Mitochondrial pyruvate carrier 2 hypomorphism in mice leads to defects in glucose-stimulated insulin secretion. Cell Rep 7: 2042-2053, 2014.

20. Maluccio $M$ and Covey A: Recent progress in understanding, diagnosing, and treating hepatocellular carcinoma. CA Cancer J Clin 62: 394-399, 2012.

21. Gluer AM, Cocco N, Laurence JM, Johnston ES, Hollands MJ, Pleass HC, Richardson AJ and Lam VW: Systematic review of actual 10-year survival following resection for hepatocellular carcinoma. HPB (Oxford) 14: 285-290, 2012.
22. Morise Z, Kawabe N, Tomishige H, Nagata H, Kawase J, Arakawa S, Yoshida R and Isetani M: Recent advances in the surgical treatment of hepatocellular carcinoma. World J Gastroenterol 20: 14381-14392, 2014.

23. Vacanti NM, Divakaruni AS, Green CR, Parker SJ, Henry RR, Ciaraldi TP, Murphy AN and Metallo CM: Regulation of substrate utilization by the mitochondrial pyruvate carrier. Mol Cell 56: 425-435, 2014

24. Gray LR, Sultana MR, Rauckhorst AJ, Oonthonpan L, Tompkins SC, Sharma A, Fu X, Miao R, Pewa AD, Brown KS, et al: Hepatic mitochondrial pyruvate carrier 1 is required for efficient regulation of gluconeogenesis and whole-body glucose homeostasis. Cell Metab 22: 669-681, 2015.

25. Ma R, Zhang W, Tang K, Zhang H, Zhang Y, Li D, Li Y, Xu P, Luo S, Cai W, et al: Switch of glycolysis to gluconeogenesis by dexamethasone for treatment of hepatocarcinoma. Nat Commun 4: 2508, 2013

26. Divakaruni AS, Wiley SE, Rogers GW, Andreyev AY, Petrosyan S, Loviscach M, Wall EA, Yadava N, Heuck AP, Ferrick DA, et al: Thiazolidinediones are acute, specific inhibitors of the mitochondrial pyruvate carrier. Proc Natl Acad Sci USA 110: 5422-5427, 2013.

27. Du J, Cleghorn WM, Contreras L, Lindsay K, Rountree AM, Chertov AO, Turner SJ, Sahaboglu A, Linton J, Sadilek M, et al: Inhibition of mitochondrial pyruvate transport by zaprinast causes massive accumulation of aspartate at the expense of glutamate in the retina. J Biol Chem 288: 36129-36140, 2013.

28. Compan V, Pierredon S, Vanderperre B, Krznar P, Marchiq I, Zamboni N, Pouyssegur J and Martinou JC: Monitoring mitochondrial pyruvate carrier activity in real time using a BRET-based biosensor: Investigation of the warburg effect. Mol Cell 59: 491-501, 2015.

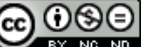

This work is licensed under a Creative Commons Attribution-NonCommercial-NoDerivatives 4.0 International (CC BY-NC-ND 4.0) License. 